\title{
Adherence Analysis of Agile Methods According to the MR-MPS Reference Model
}

\author{
Maurício Massaru Arimoto ${ }^{1}$, Edson Murakami², Valter Vieira de Camargo ${ }^{3}$, \\ Maria Istela Cagnin ${ }^{4}$
}

\author{
${ }^{1}$ Master's in Computer Science - Centro Universitário Eurípides de Marília - \\ (UNIVEM), Caixa Postal 2041 - 17.525-901 - Marília - SP - Brasil \\ ${ }^{2}$ Centro de Ciências Tecnológicas - Universidade do Estado de Santa Catarina \\ (UDESC), Caixa Postal 631 - CEP 89.223-100 - Joinville - SC - Brasil \\ ${ }^{3}$ Departamento de Computação - Universidade Federal de São Carlos (UFSCar) \\ Caixa Postal 676 - 13.565-905 - São Carlos - SP - Brasil \\ ${ }^{4}$ Departamento de Computação e Estatística - Universidade Federal do Mato Grosso do \\ Sul (UFMS), Caixa Postal 549 - 79.070-900 - Campo Grande - MS - Brasil

\begin{abstract}
In this paper some preliminary guidelines are defined to assess the adherence of agile methods to the MR-MPS model by means of established metrics, taking into consideration the intrinsic characteristics of each one. As a case study, an analysis of the adherence of three agile methods (XP, Scrum and OpenUP) according to $G$ and $F$ levels of the MR-MPS was conducted, identifying the necessary changes and their impacts. The results of this analysis show that agile methods and maturity models may be compatible, although some differences were found. In this context, adjustments are proposed in agile methods, in order to make them more adherent at the $G$ and $F$ levels of the MRMPS.
\end{abstract}

\section{Introduction}

Following the premise that software quality also depends on the quality of the process adopted in its conception, a significant number of standards and models of software maturity processes have emerged in recent years such as ISO/IEC 12207 [ISO/IEC 12207 2004], ISO/IEC 15504 [ISO/IEC 15504 2004], Capability Maturity Model Integration (CMMI) [CMMI 2006] and the MR-MPS reference model of the MPS.BR Program [Softex 2007a].

Due to constant changes in technology over the last years, new approaches to the software development have been proposed. Agile methods such as eXtreme Programming (XP) [Beck and Andres 2004], Scrum [Schwaber and Beedle 2002] and, more recently, the Open Unified Process (OpenUP) [EPF 2006, Balduino 2007] emerged as an alternative which is aimed more at micro, small and medium companies because they need to deliver quality products within a relatively short time.

One of the characteristics of agile methods is that they are focused on people and prioritize a fast supply of the system and an effective collaboration of those involved, whereas maturity models are focused on processes and prioritize a more formal and detailed documentation. Therefore, companies that use agile methods have difficulty in adjusting their methods in maturity models such as MR-MPS. Despite the many controversies concerning the compatibility between the CMMI and agile approaches, 
Glazer et. al. [Glazer et. al., 2008] show that they can be used together to achieve better results in software development.

In this paper some guidelines are provided to assess the adherence of agile methods to MR-MPS. Subsequently, a qualitative and quantitative analysis on the adherence of XP, Scrum and OpenUP in relation to G and F levels of the MR-MPS is conducted. Adjustments in agile methods are also proposals to reach the levels analyzed. The aim is to meet the profiles of micro, small and medium companies so that they can use them as a basis to seek the classification of a maturity level of the MR-MPS.

This paper is structured as follows: In Section 2, the MR-MPS model is presented. In Section 3, there is an introduction to agile methods. In Section 4, guidelines are provided to analyze the adherence of agile methods to MR-MPS. In Section 5, the study of the adherence of OpenUP, XP and Scrum according to level G of the MR-MPS is presented, together with the adjustments asked for and, in Section 6, the study is extended to level F. In Section 7, the evaluation of the results obtained are presented. In Section 8, related work is presented. Finally, in Section 9, the conclusions of the work are described.

\section{MPS.BR Program}

The MPS.BR Program has been developed since 2003, with the cooperation of various public and private Brazilian entities, co-ordinated by the Association for Promoting Brazilian Software Excellence (SOFTEX). The purpose of the program has been to define and implement a Reference Model for Improvement of Software Process (MRMPS) in all regions of Brazil and, recently, in other Latin-American countries.

The MR-MPS model has the international standards ISO/IEC 12207 and ISO/IEC 15504 as a reference and it is adhering to the CMMI-DEV (Capability Maturity Model Integration for Development) model. It is classified in sequence and cumulative maturity levels and each level contains a number of processes in a certain capability level. The maturity levels provide levels of the progress of development processes, characterizing stages of improvement in implementing the processes in the organization. A certain maturity level where the organization is, can predict its future performance in one or more processes. There are seven maturity levels: A (Optimizing), B (Quantitatively Managed), C (Defined), D (Largely Defined), E (Partially Defined), F (Managed) and G (Partially Managed) [Rocha et al. 2007, Softex 2007a]. The larger amount of levels in relation to the CMMI-DEV ensures a proper and more gradual implementation to software companies, as well as having a better visibility of the results of the process with shorter deadlines.

\section{Agile Methods}

The popularization of agile methods can be observed from 2001 with the creation of the Manifesto for Agile Software Development [Agile Manifesto 2001] when a group of researchers met each other with the aim of standardizing the various existing methods for software development [Beck and Andres 2004]. An important point of the manifesto was to define a new approach for software development, focusing on agility, flexibility, the skill to communicate and the ability to deliver new products and services with added value to the market and in a defined time. Some common principles and values that are shared by all currently agile methods were established: (i) individuals and interaction are more important than processes and tools; (ii) operating software is more important 
than detailed documentation; (iii) customers' collaboration is more important than contract negotiating; and (iv) adapting changes is more important than following a plan.

The practices they use are not new in agile methods, but the recognition that people are the main icons for the project progress and success [Highsmith and Cockburn 2001].

\section{Guidelines to Analyze the Adherence of Agile Methods for the MR-MPS Model}

An aspect that differentiates agile methods from a reference model such as MR-MPS and other conventional approaches is the granularity that some activities are carried out. Therefore, some activities can perform the same function in both agile methods and in the MR-MPS model, but with a different level of depth. Thus, a fundamental characteristic of a technique to evaluate the adherence percentage of agile methods for maturity models is to conduct a detailed analysis considering the particular characteristics of each one of them, and then propose adjustments in agile methods to meet the levels of the maturity model, without affecting the agile philosophy of these methods.

As discussed in Section 2, the MR-MPS model is structured in maturity levels. The levels contain a set of processes that must be contemplated by an organization to conform to the MR-MPS model. To investigate in detail the percentage of adherence of agile methods for certain maturity levels of the MR-MPS model and if they can work together within the same environment, a preliminary set of guidelines is established. These guidelines are based on the following assumptions: (i) the practices adopted and activities performed in agile methods are confronted with the processes (general purpose and expected results) of the maturity model. More specifically, the activities performed by a particular method are confronted with the expected results from the process of the maturity model, and (ii) the work products are equivalent in both approaches, but differ in the level of detail, as the agile methods prioritize a less detailed documentation. The assumptions are summarized using a comparative matrix shown in Table 1. The letter " $\mathrm{X}$ " at the intersection row/column means that there is a relationship among the items listed.

Table 1. Comparative matrix: agile methods X MR-MPS model

\begin{tabular}{|l|c|c|c|}
\hline \multirow{2}{*}{$\begin{array}{c}\text { Agile } \\
\text { Methods }\end{array}$} & \multicolumn{3}{c|}{ Maturity Models of the MR-MPS } \\
\cline { 2 - 4 } & $\begin{array}{c}\text { General Purpose } \\
\text { of the Process }\end{array}$ & $\begin{array}{c}\text { Expected } \\
\text { Results }\end{array}$ & $\begin{array}{c}\text { Work } \\
\text { Products }\end{array}$ \\
\hline Practices & $\mathrm{X}$ & $\mathrm{X}$ & -- \\
\hline Activities & $\mathrm{X}$ & $\mathrm{X}$ & $\mathrm{X}$ \\
\hline Work Products & -- & -- & $\mathrm{X}$ \\
\hline
\end{tabular}

Each guideline established to analyze the adherence of agile methods to MR-MPS model is described next.

\section{Guideline 1: to analyze practices and activities ${ }^{1}$}

Goal: to investigate whether the agile method, in an analysis, include practices and activities that satisfy the process of the MR-MPS at a certain maturity level.

How to apply: each process in MR-MPS is described in terms of "purpose" and "results". The purpose specifies the general aim that should be achieved with the process implementation. The expected results provide the results to be obtained with the effective process implementation. Then, from the agile method it should be checked whether their practices satisfy the process purpose of the MR-MPS. These practices can be observed

\footnotetext{
${ }^{1}$ Some agile methods use the term "tasks" instead of activities.
} 
from the activities and tasks present in the phase of the agile method lifecycle, which specify how to perform all the work. Thus, it is necessary to verify whether the execution of specified activities in the agile method in question can produce the expected results of process of the MR-MPS (Table 1). It is worth emphasizing that the necessary activities and tasks to meet the purpose and expected results are the MR-MPS user's responsibility and they are not defined in the model. The MR-MPS provides only guidance on how to achieve the expected results of the processes. It is important to assess if the activities performed in the agile method are consistent with the guidance provided by the MR-MPS and if they show the addressing of minimum requirements needed for the process implementation.

\section{Guideline 2: to analyze work products}

Aim: to investigate whether the agile method, in the analysis, addresses the minimum set of work products arising from the process implementation of the MR-MPS or provides some evidence of process addressing.

How to apply: the activities and tasks performed generally may have a work product as an output. Similarly, each expected result implementing a certain process in the MRMPS, in general, culminates with the generation of a work product, which can provide concrete evidence of the process satisfaction. The results may also be seen by a specification or a significant change of state when it runs the process. Meanwhile, it can be emphasized that the agile methods prioritize face-to-face communication and emphasize a less formal documentation and in a set of work products which are more relevant. Therefore, it is necessary to investigate the existence of work products in agile methods that address the expected results with the process implementation in analysis, more specifically, the work products to be created.

\section{Guideline 3: to apply criterion of classification}

Aim: to apply each expected result of the process implementation in an analysis in accordance with the degree of addressing achieved by the agile method.

How to apply: to assess the in-depth level that a specific expected result of each process is satisfied by the agile method, a specific criterion should be adopted, which is coherent with the Assessment Method (MA-MPS) of MPS.BR to certify the maturity level of the organization/organizational unit. Thus, in this paper a criterion for classification based on a percentage scale that ranges from 0 (Not Met) to $100 \%$ (Fully Met) was established. After classifying each expected result in its own category, metrics can be derived to calculate the percentage of expected results that are met in each process and each maturity level of the MR-MPS. From the percentage of met results a quantitative basis can be provided so that the organizations can prioritize certain processes that were not met. To classify quantitatively the degree to which the maturity levels and their process are satisfied by agile methods, criterion is defined in Table 2.

Table 2. Criterion to classify the adherence of agile methods to MR-MPS (\%)

\begin{tabular}{|l|l|}
\hline $\begin{array}{l}\text { Percentage } \\
\text { Scale }\end{array}$ & \multicolumn{1}{c|}{ Status } \\
\hline 100 & $\begin{array}{l}\text { Met (M) - when there is concrete evidence of satisfaction to the elements of the maturity } \\
\text { model }\end{array}$ \\
\hline 80 a 99 & $\begin{array}{l}\text { Largely Met (LM) - when there is concrete evidence of satisfaction to the elements of the } \\
\text { maturity model, but there is some weakness }\end{array}$ \\
30 a 79 & $\begin{array}{l}\text { Partially Met (PM) - when there is no concrete evidence of satisfaction, but there are partial } \\
\text { signs in addressing the elements of the maturity model }\end{array}$ \\
\hline 1 a 29 & $\begin{array}{l}\text { Minimally Met (MM) - when there is no concrete evidence of satisfaction, but there are } \\
\text { minimum signs in addressing the elements of the maturity model or some guidance }\end{array}$ \\
\hline 0 & $\begin{array}{l}\text { Not Met (NM) - when there is no evidence of satisfaction for the elements of the maturity } \\
\text { model }\end{array}$ \\
\hline
\end{tabular}


Guideline 4: to identify the necessary changes and assess the impact they may cause (if necessary)

Aim: to identify changes in the analyzed method and to assess the impact, in order to contemplate the requirements required to satisfy the process of the MR-MPS.

How to apply: from the analysis, the necessary changes to minimize the potential problems identified in the agile method in relation to the maturity model should be identified and proposed, where the status is classified as "LM", "PM", "MM" or "NM". The adaptive approach adopted by agile methods, unlike the conventional, is ideal for environments whose characteristics are requirements which are often changing, making the changes easier. However, any change must comply with the values and principles established by methods so that organizations can use them effectively and, mostly, not to affect the agile philosophy of the methods. Moreover, the adjustments proposed need to take into consideration the type of organization and the particular characteristics of each project. This includes the business aim, the size and team's experience, and other relevant issues. Subsequently, it is important to consider whether the changed method remains "agile". Considering this, we suggest checking whether the agile method which was changed does not "hinder" the twelve principles of the Agile Manifesto.

The systematic application of the guidelines is represented by the algorithm in Figure 1. The application process is sequential, and guidelines 1, 2 and 3 should be executed together, since they are closely linked. A suggestion is to start by the lowest level of the maturity model, although it is not necessary.

- For each LEVEL of the Maturity Model

- to apply GUIDELINE 1 for each process of level of the maturity model in question

- to apply GUIDELINE 2 for each process of level of the maturity model in question

- to apply GUIDELINE 3 for each process of level of the maturity model in question

- If status of adherence of Agile Method <> "M"

- to apply GUIDELINE 4 for each process of level of the maturity model in question

End of algorithm

Figure 1. Algorithm of application of guidelines

\section{Adherence Analysis of Agile Methods to Level G of the MR-MPS}

In this section, the adherence analysis of agile methods XP, Scrum and OpenUP in relation to the maturity level $G$ of the MR-MPS is presented, following the guidelines established in Section 4. Please note that the algorithm is applied only in the initial levels of the MR-MPS (G and F).

Level $\mathbf{G}$ is the initial phase of implementation of the MR-MPS and therefore it tends to cause significant changes in the organizational culture of the company, requiring careful planning in its implementation. This level focuses on the partial management of software development projects and includes two processes: Project Management (PM) and Requirement Management (RM). The processes addressed in their maturity levels and the expected results with the processes implementation are described in detail in the MPS.BR Implementation Guide [Softex 2007b]. Next, a study to examine in detail the 
adherence of OpenUP in relation to PM and RM processes is presented, based on guidelines established in Section 4. The detailed analysis of adherence of other agile methods studied (XP and Scrum) is not presented in this paper due to a shortage of space. Nevertheless, the obtained results are shown at the end of this section.

\subsection{Application of the Algorithm of Adherence Analysis}

\section{To analyze practices and activities}

The purpose of the $\mathbf{R M}$ process is to manage the product requirements and the product components of the project and identify inconsistencies among the requirements, the project plans and the work products of the project. The OpenUP establishes a series of disciplines to address the various aspects of software development. Each discipline includes a set of tasks and steps to be taken towards achieving the aims, culminating with the production and/or modification of a work product. The Requirements discipline, for example, covers the major aims of the RM process and defines the minimum tasks necessary to extract, analyze, specify, validate and manage the requirements for the system to be developed. The implementation of RM process addresses a set of five expected results (represented by RM1 to RM5). The following are the expected results considered most critical.

The RM3 refers to the bi-directional tracking between requirements and work products. Both in the OpenUP and as in the XP and Scrum, the techniques involved in the software development are not so important, but the end result is. Thus, the OpenUP does not define mechanisms to track requirements and work products, and there is no evidence in the OpenUP that meet this purpose. Therefore, this result is considered Not Met.

The purpose of the PM process is to identify, establish, coordinate and monitor the activities, tasks and resources that a project needs to generate a product and/or service, in the context of the requirements and project restriction [ISO/IEC 12207 2004]. The implementation of the PM process addresses a set of seventeen expected results (represented by PM1 to PM17), discussed below, emphasising the expected results which are more critical.

The PM7 refers to the human resources planning. In the OpenUP, the function (roles) and responsibilities are assigned in accordance with the knowledge and skills of each team member, as part of the project planning. However, it is not an explicitly defined plan for the team training, which must integrate the human resources planning. Therefore, this result is classified as Partially Met. The PM8 refers to the planning of tasks, resources and work environment. The OpenUP tasks and resources are defined in the project plan and iteration plan. Meanwhile, plans for environments and special resources such as tools, equipment and travel are not explained and thus this result is Partially Met. The PM9 refers to the planning of relevant data for the project. In the OpenUP, this data are gathered in various artifacts (project plan, iteration plan, work items list, etc.), reports, lessons learned, among others. The distribution can be given in printed form, email or web publishing. However, the OpenUP makes no mention of issues of privacy and data security and it is considered as a weak point. Thereby, this result is classified as Largely Met.

\section{To analyze work products}

In Table 3 there is a correlation between the typical work products in the MR-MPS and those found in the OpenUP. Each work product is discriminated in terms of what expected result it comes from (RM1 to RM5 and PM1 to PM17). For example, to 
completely satisfy the expected result \#1 and \#2 for the RM process are required welldefined criteria to evaluate and approve requirements and can be carried out in meetings. Similarly, in the OpenUP the most common way to assess and approve the requirements is by meetings (formal or informal), where the requirements are reviewed with stakeholders to ensure consistency, to assess quality and identify the necessary changes. The purpose is to ensure that the requirements are complete, clear, consistent, and atomic and are feasible to implement and test. The checklist also supports the criteria for evaluation and requirement approval.

Table 3. Analysis of work products at level $\mathrm{G}$

\begin{tabular}{|c|c|c|c|}
\hline & & MR-MPS & OpenUP \\
\hline \multirow{5}{*}{$\sum_{\Upsilon 1}$} & 1 & $\begin{array}{l}\text { Criteria for Evaluation and Acceptance of } \\
\text { Requirements/Requirements Document }\end{array}$ & $\begin{array}{l}\text { Face-to-face Meetings/System-Wide } \\
\text { Requirements/Use-Case Model/Checklist }\end{array}$ \\
\hline & 2 & $\begin{array}{l}\text { Approval of Requirements (Kick off Meetings) } \\
\text { /Requirement Impact Assessment }\end{array}$ & Face-to-face Meetings/Checklist \\
\hline & 3 & $\begin{array}{l}\text { Tracking System of Requirements and Work } \\
\text { Products /Traceability Matrix of Requirements } \\
\text { and Work Products }\end{array}$ & -- \\
\hline & 4 & $\begin{array}{l}\text { Results of Revisions/Documentation of } \\
\text { Inconsistencies/Corrective Actions }\end{array}$ & Record in the Iteration Plan/Work Items List \\
\hline & 5 & $\begin{array}{l}\text { Requirements Status/Requirements } \\
\text { Repository/Decision History about the } \\
\text { Requirements }\end{array}$ & Work Item List \\
\hline \multirow{14}{*}{$\sum_{n}$} & 1 & $\begin{array}{l}\text { Work Breakdown Structure (WBS) or Vision } \\
\text { Document }\end{array}$ & Vision Document \\
\hline & 2 & Estimate of Size & $\begin{array}{l}\text { Agile Estimate of Size (typically measured } \\
\text { using a neutral unit such as points) }\end{array}$ \\
\hline & 3 & Project Lifecycle Phases & $\begin{array}{l}\text { Project Lifecycle Phases (specified in the } \\
\text { Project Plan) }\end{array}$ \\
\hline & 4 & $\begin{array}{l}\text { Project Effort Estimates/ } \\
\text { Project Cost Estimates }\end{array}$ & $\begin{array}{l}\text { Typically Using the Units of Actual Days or } \\
\text { Actual Hours /Multiply the Approximate Cost } \\
\text { per Person by the Size of an Iteration }\end{array}$ \\
\hline & 5 & $\begin{array}{l}\text { Project Schedule } \\
\text { and Budget }\end{array}$ & $\begin{array}{l}\text { Project Schedule and Budget (specified in the } \\
\text { Iteration Plan and Project Plan) }\end{array}$ \\
\hline & 6 & Risk Management Plan & $\begin{array}{l}\text { Identified Risks/Risk Impact and Probability of } \\
\text { Occurrence (specified in the Project Plan or } \\
\text { Iteration Plan)/Risk List }\end{array}$ \\
\hline & 7,8 & Resource Management Plan & Project Plan/Iteration Plan/Work Items List \\
\hline & 9 & Data Management Plan & Iteration Plan \\
\hline & 10 & Overall Project Plan & Project Plan \\
\hline & 11,12 & $\begin{array}{l}\text { Feasibility Study/ Review and Commitment to } \\
\text { the Project Plan }\end{array}$ & Project Planning/ Face-to-Face Meetings \\
\hline & 13 & Evaluation of Project Progress/Project Status & $\begin{array}{l}\text { Iteration Plan/Reports } \\
\text { and project burndown) }\end{array}$ \\
\hline & 14 & Communication Management Plan & Iteration Plan/Project Plan \\
\hline & 15 & $\begin{array}{l}\text { Documented Results of Project Milestone } \\
\text { Review }\end{array}$ & Documented in the Iteration Plan \\
\hline & 16,17 & $\begin{array}{l}\text { Record of Problems and Monitoring of } \\
\text { Corrective Actions/Corrective Actions Plan }\end{array}$ & Record in the Work Items List/Iteration Plan \\
\hline
\end{tabular}

\section{To apply criterion of classification}

In Table 4, each expected result implementing the RM process is categorized according to the criterion proposed in Section 4 (Table 2), i.e., Met (M), Largely Met (LM), Partially Met (PM), Minimally Met (MM) and Not Met (NM). The results regarding the PM process are presented in Table 5. It can be observed that both tables include XP and Scrum that have been evaluated following the same procedures of OpenUP, as reviewed previously.

From the total count of expected results in the implementation of each process by each agile method studied, the percentage of expected results can be calculated (which are M, LM, PM, MM and NM). The explanation of the results for the RM and PM 
processes of level $\mathrm{G}$ can be viewed in Table 6. The adherence percentage of agile methods in relation to each process was calculated dividing the sum of the expected results in each category (which may be M, LM, PM, or NM) by the sum of expected results of each process (RM or PM). As illustrated, the most expected results fit in the "Met" category. This implies that there are no necessary adjustments in the methods analyzed. Additionally in Table 7, the explanation of the results for level G of the MRMPS is presented, which was calculated using the previous procedure, but considering the sum of the RM and PM processes together. Among the agile methods considered, the OpenUP had a better use, reaching $81.9 \%$ of adherence. The majority of the expected results met the PM process.

Table 4. Classification of expected results of Requirement Management Process

\begin{tabular}{|c|c|c|c|c|c|}
\hline \multirow{2}{*}{$\begin{array}{c}\text { Agile } \\
\text { Methods }\end{array}$} & \multicolumn{4}{|c|}{ Expected Results - Requirement Management } \\
\cline { 2 - 6 } & 1 & 2 & 3 & 4 & 5 \\
\hline XP & $\mathrm{M}$ & $\mathrm{M}$ & $\mathrm{NM}$ & $\mathrm{M}$ & $\mathrm{M}$ \\
\hline Scrum & $\mathrm{M}$ & $\mathrm{M}$ & $\mathrm{NM}$ & $\mathrm{M}$ & $\mathrm{LM}$ \\
\hline OpenUP & $\mathrm{M}$ & $\mathrm{M}$ & $\mathrm{NM}$ & $\mathrm{M}$ & $\mathrm{M}$ \\
\hline
\end{tabular}

Table 5. Classification of expected results of Project Management Process

\begin{tabular}{|c|c|c|c|c|c|c|c|c|c|c|c|c|c|c|c|c|c|}
\hline Agile & \multicolumn{17}{|c|}{ Expected Results - Project Management } \\
\hline Methods & 1 & 2 & 3 & 4 & 5 & 6 & 7 & 8 & 9 & 10 & 11 & 12 & 13 & 14 & 15 & 16 & 17 \\
\hline $\mathrm{XP}$ & $\mathrm{M}$ & $\mathrm{M}$ & $M$ & LM & $\mathrm{PM}$ & MM & $\bar{M}$ & $\mathrm{M}$ & $M$ & $\mathrm{M}$ & $\mathrm{M}$ & $\mathrm{M}$ & $\mathrm{M}$ & $\mathrm{M}$ & $M$ & $\mathrm{PM}$ & $\mathrm{M}$ \\
\hline Scrum & $\mathrm{M}$ & NM & $\mathrm{M}$ & LM & $\mathrm{M}$ & MM & $\mathrm{M}$ & $\mathrm{M}$ & PM & $\mathrm{M}$ & $\mathrm{M}$ & $\mathrm{M}$ & $\mathrm{M}$ & $\mathrm{M}$ & $\mathrm{M}$ & $\mathrm{M}$ & PM \\
\hline OpenUP & $\mathrm{M}$ & $\mathrm{M}$ & $\mathrm{M}$ & $M$ & $\mathrm{M}$ & $\mathrm{M}$ & PM & PM & LM & $\mathrm{M}$ & $\mathrm{M}$ & $\mathrm{M}$ & $\mathrm{M}$ & $\mathrm{M}$ & $\mathrm{M}$ & $\mathrm{M}$ & $\mathrm{M}$ \\
\hline
\end{tabular}

Table 6. Percentage of adherence of agile methods for processes of level G (\%)

\begin{tabular}{|l|c|c|c|c|c|c|}
\hline Agile Methods & \multicolumn{2}{|c|}{ XP } & \multicolumn{2}{c|}{ Scrum } & \multicolumn{2}{c|}{ OpenUP } \\
\hline Status / Processes & PM & RM & PM & RM & PM & RM \\
\hline Met & 76.5 & 80 & 70.6 & 60 & 82.4 & 80 \\
\hline Largely Met & 5.9 & - & 5.9 & 20 & 5.9 & - \\
\hline Partially Met & 11.7 & - & 11.7 & - & 11.7 & - \\
\hline Minimally Met & 5.9 & - & 5.9 & - & - & - \\
\hline Not Met & - & 20 & 5.9 & 20 & - & 20 \\
\hline
\end{tabular}

Table 7. Percentage of adherence of agile methods to level G (\%)

\begin{tabular}{|l|c|c|c|}
\hline \multicolumn{1}{|c|}{ Status } & XP & Scrum & OpenUP \\
\hline Met & 77.4 & 68.2 & 81.9 \\
\hline Largely Met & 4.5 & 9.1 & 4.5 \\
\hline Partially Met & 9.1 & 9.1 & 9.1 \\
\hline Minimally Met & 4.5 & 4.5 & - \\
\hline Not Met & 4.5 & 9.1 & 4.5 \\
\hline
\end{tabular}

\section{To identify necessary changes and assess the impact they may cause}

From the analysis conducted, some discrepancies were noted between agile methods and the maturity model. The adjustments proposed for the problems identified in agile methods analyzed (including XP and Scrum) are presented below. These adjustments are in accordance with agility principles established in Agile Manifesto and the principles and practices intrinsic of each method, causing little impact on effort, cost and quality in an agile software development process, as desired.

\subsection{Adjustments Proposed}

From the perspective of the RM process, the adjustments aim to minimize the shortage of traceability mechanism of requirements and work products and a lack of history changes in the requirements, following the guidelines of the MR-MPS, namely: (i) to use techniques based on cross-references (for example traceability matrix) that can be generated in a spreadsheet or with the aid of a specific tool; and (ii) to use a document to 
add new requirements or change the existing ones. Recording the requests for changes to a history of decisions in the requirements is available. A report on the impact analysis of decisions should also be prepared.

From the perspective of the PM process, the adjustments proposed in agile methods aim to mainly minimize the problems related to the lack of estimating size and cost, risk management, training, special resources, problems and dependencies registration and corrective action management. These adjustments are listed below: (i) to follow the technique of agile size estimate used in the OpenUP as a basis, which generally uses relative measures called points or story points and planning poker suggested by Cohn [2006]; (ii) to maintain in a spreadsheet all the costs for the project (salaries, training, travel, among others) so that these data can be used in a budget; (iii) based on the risks management of the OpenUP, it is important to elaborate a list containing the risks which are more common and, as appropriate, to identify potential risks inherent in the project, verifying the probability of occurrence (low, medium, high), category (technical, managerial, organizational, external), impact (light, medium, hard), magnitude (combination of probability and impact of risk) and priority treatment for each one. This list can be prepared in a spreadsheet; (iv) to define the training necessary for project run (courses, workshops etc.) during the project planning task and to document in the Project Plan; (v) to use a spreadsheet to record all the special resources or to use the Project Plan to store these pieces of information; (vi) to use a spreadsheet, a tool as bugtracking or another mechanism for problem management, so that they can be analyzed, recorded and tracked and to provide control over the actions taken, responsible for actions and results.

\section{Adherence Analysis of Agile Methods to Level F of the MR-MPS}

Level $\mathbf{F}$ corresponds to the second phase of implementation of the MR-MPS and its focus is to aggregate processes that support the project management with regards to quality assurance and measurement, as well as those who organize the work products using the configuration management. Therefore, the following processes should be addressed: Measurement (ME), Quality Assurance (QA), Configuration Management (CM) and Acquisition (AC). The processes contemplated in their maturity levels and the expected results with the process implementation are described in detail in MPS.BR Implementation Guide, Part 2: Level F [Softex 2007b].

A detailed analysis of the adherence of the OpenUP in relation to processes of level $\mathrm{F}$ is presented next. It should be noted that other agile methods were also analyzed, but do not appear due to the shortage of space. Nevertheless, the obtained results are presented at the end of this section.

\subsection{Applying the Algorithm of Adherence Analysis}

\section{Analysing practices and activities}

The purpose of the ME process is to collect, analyze and disseminate data related to developed products and the processes which were implemented in the organization and in its projects in order to support the organizational objectives [Softex 2007b]. Its implementation should consist of a set of seven expected results (represented by ME1 to ME7). The following is an analysis of the most critical expected results.

The ME1 refers to the measurement aims. In the OpenUP, the measurement aims are defined and maintained in order to assist decision-making based on the analysis 
results made in the planning stages of the project. Meanwhile, a weakness identified is that there is no method for setting objectives and therefore this result is considered to be Largely Met. The ME2 refers to the definition of a set of measures. In the OpenUP, the set of measures is defined and prioritized during the Iteration Planning. However, the OpenUP does not specify clearly how to store, document, review and update measures. Thus, it is considered that this result is Partially Met. The ME3 refers to the procedures for collection and storage measures. In the OpenUP, the procedures for the collection of measures are specified in the Project Plan and/or Iteration Plan. Nevertheless, procedures for storage are not explained. Therefore, it is considered that this result is Partially Met. The ME6 refers to the data storage and analysis results. In the OpenUP, the data storage is part of the tasks of planning the project and iteration. However, the OpenUP does not specify mechanisms to store the results of the analysis carried out. Therefore, this result is Partially Met.

The purpose of the QA process is to ensure that the work products and the respective processes are in accordance with the plans and resources defined and that they address a set of four expected results (represented by QA1 to QA4) with the process implementation [Softex 2007b]. The following is the analysis of the most critical expected results.

The QA1 and QA2 refer to objective evaluations of products and processes. In the OpenUP, during the task of Evaluation Results near the iteration end, the team should jointly evaluate if the aims and evaluation criteria established in Iteration Plan have been achieved, and if the team carried out the plan and concluded all the work items as planned. Nevertheless, both the results suggest independent audits to evaluate products and processes objectively and do not accept assessments by the team. Therefore, these results are classified as Minimally Met.

The purpose of the $\mathbf{C M}$ process is to establish and maintain all work products of a process or project consistently and make them available to all those interested [Softex 20007b]. Its implementation includes a set of seven expected results (represented by CM1 to CM7).

In the OpenUP, as well as $\mathrm{XP}$ and Scrum, CM activities are allocated to organizations. The OpenUP does not define a system configuration management, although there are some practices that help in CM activities and make an implicit control possible, such as small releases and continuous integration. The OpenUP considers only the primary items inherent in the system construction, such as source code and the executable version of the system. The OpenUP does not also define a criterion to select the configuration items and baselines and does not mention how it should be done. Another aspect observed is that there is no mechanism to keep the configuration items and baselines under control, so that these items can be accessed and earlier versions can be retrieved when needed. Configuration Audit is also not considered. In addition, the storage, handling and release of the configuration items and baselines are not explicitly controlled. Therefore, none of the expected results of the CM process are classified as Met.

The aim of the AQ process is to fundamentally assist the organizations that required acquiring new software products and related services, supported by MR-MPS. It should be noted that agile methods do not establish policies for the purchase software products and related services. This process is beyond the scope of agile methods, and is seen as complementary and its implementation therefore depends on the needs of each organization. 


\section{To analyze work products}

In Table 8, the correlation between work products of level $\mathrm{F}$ of the MR-MPS and the OpenUP is presented. The purpose was to verify in the OpenUP the existence of work products that meet the requirements of each expected result of the process implementation (second column on the left). In item CM5, for example, the OpenUP only deals with the change request management using the artifact Work Item List.

Table 8. Analysis of work products at level $\mathbf{F}$

\begin{tabular}{|c|c|c|c|}
\hline & \multicolumn{2}{|c|}{ MR-MPS } & \multirow{2}{*}{$\begin{array}{l}\text { OpenUP } \\
\text { Project Plan/Iteration Plan }\end{array}$} \\
\hline \multirow{5}{*}{ Ш } & 1 & Measurement Objectives & \\
\hline & 2 & Measure Specifications & Iteration Plan/Work Items List \\
\hline & 3,4 & $\begin{array}{l}\text { Measure Collection and Storage Procedures, Documentation } \\
\text { and Revision of Selected Measure /Analysis Specifications } \\
\text { and Procedures/Measure Analysis and Collection Tools }\end{array}$ & Project Plan/Iteration Plan \\
\hline & 5,6 & $\begin{array}{lcccc}\text { Measurement } & \text { Data Sets/Analysis } & \text { Results and } & \text { Draft } \\
\text { Reports/Store Data Inventory } & & & \\
\end{array}$ & Project Plan/Iteration Plan \\
\hline & 7 & Communication of Analysis Results & Reports and Graphics \\
\hline \multirow[t]{2}{*}{ 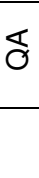 } & 1,2 & $\begin{array}{l}\text { Quality Audits } \\
\text { Evaluation Reports/Noncompliance Reports/ Corrective } \\
\text { Actions }\end{array}$ & $\begin{array}{l}\text {-- } \\
\text { Iteration } \\
\text { Reports }\end{array}$ \\
\hline & 3,4 & $\begin{array}{l}\text { Documentation of Problems and Noncompliance/Corrective } \\
\text { Actions Reports/Evaluation Reports }\end{array}$ & Work Items List \\
\hline \multirow{7}{*}{$\sum_{0}$} & 1 & $\begin{array}{l}\text { Configuration Management System/Access Control } \\
\text { Procedures/Change Request Database }\end{array}$ & -- \\
\hline & 2 & Identified Configuration Items/Configuration Management Plan & -- \\
\hline & 3 & Creation of Baseline/Archives of Baselines & -- \\
\hline & 4 & Status of Configuration Items and Baselines & -- \\
\hline & 5 & $\begin{array}{l}\text { Changes in Control and Revision History of Configuration } \\
\text { Items/ Archives of Baselines }\end{array}$ & Work Items List \\
\hline & 6 & Configuration Audits Results/Action Items & -- \\
\hline & 7 & $\begin{array}{l}\text { Configuration Items and baselines Inventory/ Baselines } \\
\text { Delivery Control Procedures }\end{array}$ & -- \\
\hline
\end{tabular}

\section{Applying the classification criterion}

In Table 9, the classification of expected results of the implementation of each process of level $\mathrm{F}$ is presented. As performed at level $\mathrm{G}$, each expected result was classified according to criterion established in Section 4 (Table 2). It can be observed in level F that the number of results " $M$ " is much smaller in relation to level $G$. The coverage percentage of each method in relation to processes of level $\mathrm{F}$ is shown in Table 10. The explanation of the results for level $F$ is illustrated in Table 11. Unlike level $G$, approximately $47 \%$ of the results were Not Met (NM). This occurred due to the lack of the configuration management system and lack of policies towards the acquisition of software products and related services, which are not prioritized in agile methods. By disregarding the AQ process from the analysis, the adherence percentage for the three agile methods (results completely met) should all increase by about $50 \%$.

Table 9. Classification of expected results of processes of level $\mathbf{F}$

\begin{tabular}{|c|c|c|c|c|c|c|c|c|c|c|c|c|c|c|c|c|c|c|c|}
\hline \multirow{2}{*}{$\begin{array}{c}\text { Agile } \\
\text { Methods }\end{array}$} & \multicolumn{7}{|c|}{ ME } & \multicolumn{4}{|c|}{ QA } & \multicolumn{7}{|c|}{$\mathrm{CM}$} & \multirow{2}{*}{$\begin{array}{l}A Q \\
1-9 \\
\end{array}$} \\
\hline & 1 & 2 & 3 & 4 & 5 & 6 & 7 & 1 & 2 & 3 & 4 & 1 & 2 & 3 & 4 & 5 & 6 & 7 & \\
\hline$X P$ & LM & LM & PM & $M$ & $M$ & LM & $M$ & MM & MM & LM & $M$ & NM & PM & MM & NM & PM & NM & NM & NM \\
\hline Scrum & PM & PM & LM & $M$ & $M$ & MM & $M$ & MM & MM & $M$ & PM & NM & PM & $\mathrm{MM}$ & NM & PM & NM & NM & NM \\
\hline OpenUP & LM & PM & PM & $M$ & $M$ & PM & $M$ & MM & MM & $M$ & $M$ & NM & PM & MM & NM & PM & NM & MM & NM \\
\hline
\end{tabular}

Table 10. Percentage of adherence of agile methods for processes of level $F(\%)$

\begin{tabular}{|l|c|c|c|c|c|c|c|c|c|c|c|c|}
\hline & \multicolumn{3}{|c|}{ ME } & \multicolumn{2}{|c|}{} & \multicolumn{2}{c|}{ QA } & \multicolumn{2}{|c|}{ CM } & \multicolumn{3}{|c|}{ AQ } \\
\hline & XP & Scrum & OpenUP & XP & Scrum & OpenUP & XP & Scrum & OpenUP & XP & Scrum & OpenUP \\
\hline M & 42.8 & 42.8 & 42.8 & 25 & 25 & 50 & - & - & - & - & - & - \\
\hline LM & 42.8 & - & 14.4 & 25 & - & - & - & - & - & - & - & - \\
\hline PM & 14.4 & 42.8 & 42.8 & 25 & 50 & 25 & 28.6 & 28.6 & 28.6 & - & - & - \\
\hline MM & - & 14.4 & - & 25 & 25 & 25 & 14.3 & 14.3 & 28.6 & - & - & - \\
\hline NM & - & - & - & - & - & - & 57.1 & 57.1 & 42.8 & 100 & 100 & 100 \\
\hline
\end{tabular}


Table 11. Percentage of adherence of agile methods to level $F(\%)$

\begin{tabular}{|l|c|c|c|}
\hline \multicolumn{1}{|c|}{ Status } & XP & Scrum & OpenUP \\
\hline Met & 14.8 & 14.8 & 18.5 \\
\hline Largely Met & 14.8 & 3.7 & 3.7 \\
\hline Partially Met & 11.1 & 18.5 & 18.5 \\
\hline Minimally Met & 11.1 & 14.8 & 14.8 \\
\hline Not Met & 48.2 & 48.2 & 44.5 \\
\hline
\end{tabular}

\section{To identify necessary changes and assess the impact they may cause}

Based on the analysis results, it was observed that agile methods do not fully meet a large percentage of the expected results when implementing processes addressed in level $\mathrm{F}$. The solutions to the problems identified are presented in the next section.

\subsection{Adjustments Proposed}

The adjustments proposed in the agile methods to make them compatible with ME process are listed below and the aim is to minimize the problems mainly related to defining the measurement and procedures for the documentation, collection and storage of the measures, namely: (i) to use the method Goal Question Metrics (GQM) proposed by Basili and Weiss [Solingen and Berghout 1999] to assist in establishing measurement objectives or other methods available; (ii) to bring together a set of measures in a document that can be identified by name, measure unit and description. The document should be reviewed by the top manager and updated as necessary. The measures can be classified as basic or direct (obtained from observable attributes, usually determined by counting) and derived or indirect (obtained from observable attributes, but which are calculated by the combination of other measures); (iii) to establish well-defined criteria to properly collect and store the measures, if possible, using a specific tool for that. The frequency with which the measures are collected, those responsible for collection, storage, recovery and data security and the necessary tools should be specified; (iv) to create a repository for storing the measures, so that they can be easily accessed and available for future use.

The adjustments proposed in agile methods to make them compatible with the QA process, minimizing the problems related to the objective evaluation of products and processes and recording problems and nonconformance are presented as follows: (i) to define a group responsible for quality assurance activities and promote independent audits to assess the quality of products and processes; (ii) to use a spreadsheet or automated tool to record and monitor the correction of problems and nonconformance and to communicate to those who are interested.

Finally, the adjustments to the problems identified in agile methods according to the CM process are presented as follows: (i) to define a system of configuration management (version control system, change control system and construction management system) so that the configuration items can be identified and managed; (ii) to define someone or a group responsible for the configuration management, configuration items and baselines; (iii) to define criteria to identify configuration items and baselines based on, for example: items that define scope and related items, items used frequently, among others; (iv) to use tools for control versions so that the changes can be managed in a systematic manner, subjected to the system configuration management and make them available to all those who are interested; (v) to promote management configuration audits to evaluate, review and approve all requests of changes; (vi) to use in an effective way the system of configuration management defined for the project to ensure the integrity of configuration items and baselines. 


\section{Data Analysis}

From the percentage obtained in each process in the respective maturity level, the percentage scale defined in Table 2 (Section 4) can be used to rank in quantitative terms how much a particular process or maturity levels are satisfied by agile methods. This classification provides a quantitative view regarding which processes a company should concentrate its efforts to improve them and thus be classified into a certain maturity level of the MR-MPS.

The final results of the adherence of agile methods to levels $G$ and $F$ of the MRMPS are presented in Table 12. It should be mentioned that the study on the adherence of XP and Scrum in relation to levels $G$ and $F$ of the MR-MPS was also conducted following the same rigor, based on guidelines established in Section 4. Among the expected results in their respective maturity levels, most are in the Partially Met (PM) and Minimally Met (MM) categories, emphasizing the need for adjustments in agile methods to minimize the problems encountered.

Table 12. Classification of adherence of agile methods to levels $G$ and $F$ of the MR-MPS

\begin{tabular}{|c|c|c|c|}
\hline Agile Methods & OpenUP & Scrum & XP \\
\hline Level G & LM & PM & PM \\
\hline Level F & M & MM & MM \\
\hline
\end{tabular}

In short, the agile methods analyzed meet a large percentage of the expected results of processes addressed in level G, highlighting the OpenUP. Nevertheless, it can be seen that they do not prioritize techniques for tracking requirements and work products, such as the guidelines of MR-MPS to meet the RM process of level G. These methods emphasise fast delivery of versions of the software and the final product and not techniques to be used in development. At level $\mathrm{F}$, configuration management activities are allocated to organizations and are not explicitly defined, and quality audits to assess products and processes objectively are not either. Moreover, the AQ process is not addressed but can be excluded from the MPS.BR assessment if it does not belong to the scope of the project.

An important aspect is related to risk managing. OpenUP, unlike XP and Scrum, provides a framework to risk management, setting milestones for risk mitigation and prioritizing their reduction during the initial stages and adding value throughout the lifecycle of the project. The risks should be reviewed regularly and the strategies for its reduction should be implemented strictly. Another aspect observed is that many of the problems identified in agile methods are related to the lack of documentation as in the agile view, the running of software is more important than a detailed documentation. This problem is minimized with the inclusion of the items which are more relevant to the project development, avoiding unnecessary documentation.

\section{Related Work}

In the literature, there are some studies that compare agile methods with maturity models, more specifically the CMM models. Paulk [Paulk 2001] compared XP with CMM, taking into account only those components with the highest level (Key Process Areas - KPA's and aims), without going into details of practices and sub-practices of the model. The author observed that some areas of the CMM process were partially met by XP and others were not addressed. Nevertheless, according to Paulk, XP incorporates good practices of Software Engineering which, when implemented carefully in an appropriate environment, covers many practices related to levels 2 and 3 of CMM.

Turner and Jain [Turner and Jain 2002] conducted a study on the adequacy of the CMMI and agile methods and they reported that the approaches have some similarities, 
however $42 \%$ of the areas of the CMMI process are in conflict with the practices adopted by agile methods. Although there were differences, the authors have concluded that they can be used within the same environment since they adopt appropriate policies in the organizational development process. In the study, the authors have not mentioned any specific agile method.

There is still no consensus about compatibility between agile methods and maturity models. Recently, Glazer et al. [Glazer et al., 2008] conducted a study in order to clarify the divergences of agile methods and CMMI that should not exist. Despite the differences, the authors propose to use both as they can bring many benefits for software development, aggregating value to business.

To analyse agile methods from the perspective of the MR-MPS model, which is the focus of this work, Santana, Timóteo and Vasconcelos [Santana, Timóteo and Vasconcelos 2006] have presented a mapping MR-MPS with the XP, considering only levels $\mathrm{G}$ and $\mathrm{F}$. The authors have presented some suggestions for necessary changes to ensure that a company using XP can fit in at levels $G$ and $F$ of the MR-MPS. Nevertheless, in the work it is not specified how the XP meets the processes and the expected results by implementing processes in the levels analyzed. Moreover, in the work only the agile XP method is used as a basis for study.

In this context, it is important to use other agile methods to analyse the compatibility to the MR-MPS in order to constitute a solid basis so that organizations can choose among the existing agile methods, the most appropriate for its purpose. Another important point refers to the procedure that should be adopted to analyze the compatibility of agile methods according to the MR-MPS model. In the literature, there is a shortage of work that give guidelines to conduct the adherence of agile methods in relation to the MR-MPS in a qualitative and quantitative way.

\section{Final Remarks}

The main aim of this paper was to provide a preliminary set of guidelines to investigate the adherence of agile methods to the MR-MPS model in order to serve as a guide for both researchers and companies interested in using agile methods and maturity models within the same environment.

As a case study, an analysis of the adherence of agile methods XP, Scrum and OpenUP to MR-MPS was conducted, considering the intrinsic characteristics of each one. From the analysis, we have identified some conflicts and differences among the approaches. In this context, adjustments were proposed in the agile methods analyzed in order to make them more compliant to levels G and F of MR-MPS, without changing the philosophy. These adjustments were planned to be as generic as possible and consistent with agile methods and maturity models. Therefore, MR-MPS guidelines were followed, as well as the principles, practices and values adopted by the agile methods. It is hoped that this study will serve as a support for different organizations that use agile methods and would like to fit into maturity levels of the MR-MPS.

Despite having a different approach, it can be inferred that agile methods and maturity models such as the MR-MPS can be used together, thereby exploring the benefits and complementary aspects of both approaches. However, it is important to point out that changes in agile methods must be implemented in accordance with the organization and the development project in question in order to reduce their impact and meet the different interests and business aim. These changes must be planned and managed until completed. 
The adjustments proposed in this paper will be made valid in a more in-depth case study which is being conducted with the OpenUP in an industrial environment to obtain consolidated results. Furthermore, the guidelines established will also be used to assess the adherence of agile methods according to other maturity levels of the MR-MPS and will be refined if necessary.

\section{Acknowledgements}

The authors would like to thank CAPES (Coordenação de Aperfeiçoamento de Pessoal de Nivel Superior) for the financial support.

\section{References}

Agile Manifesto. (2001), "Manifesto for Agile Software Development", http://www.agilemanifesto.org.

Balduino, R. (2007), “Introduction to OpenUP”, http://www.eclipse.org/epf/general/ openup.pdf.

Beck, K. and Andres, C. (2004), "Extreme Programming Explained: embrace change”. AddisonWesley, $2^{\text {nd }}$ edition.

"CMMI® for Development" (version 1.2). (2006), Technical Report CMU/SEI-2006-TR-008, PA: Software Engineering Institute, Carnegie Mellon University, August.

Cohn M. (2006), “Agile Estimating and Planning”. Prentice Hall.

Eclipse Process Framework (EPF). (2006), "Open Unified Process (OpenUP)".

Glazer, H. et al. (2008), "CMMI or “Agile: why not embrace both!" Technical Report CMU/SEI2008-TN-003, Software Engineering Institute, Carnegie Mellon University, November.

Highsmith, J. and Cockburn, A. (2001), "Agile Software Development: the Business of Innovation". IEEE Computer Society, September, p. 20-122.

ISO/IEC 12207: 1995/Amd 1: 2002/Amd 2: 2004. Information Technology - Software Life-cycle Processes.

ISO/IEC 15504. Information Technology - Process Assessment. Part 1 - Concepts and Vocabulary; Parte 2 - Performing an Assessment; Part 3 - Guidance on Performing an Assessment; Part 4 - Guidance on use for Process Improvement and Process Capability Determination; and Part 5 - An Exemplar Process Assessment Model.

Kroll, P. (2006), "Who will benefit from the Eclipse Process Framework". Eclipse Process Framework, Eclipse Foundation.

Paulk, M.C. (2001), "Extreme Programming from CMM Perspective", IEEE Software, November, p. 1-8.

Rocha, A.R., et al. (2007), Nationwide Program for Software Process Improvement in Brazil. In: Proceedings of the $6^{\text {th }}$ International Conference on the Quality of Information and Communications Technology (QUATIC'2007) (Lisbon New University, Lisbon, Portugal, Sep. 12-14, 2007), p. 449-460.

Santana, C.A., Timóteo, A.L. and Vasconcelos, A.M.L. (2006), Mapping of the MPS.BR Model for Companies that use XP as Development Methodology. In: V Simpósio Brasileiro de Qualidade de Software (SBQS'2006) (Espírito do Santo, Brasil, 2006), p. 130-146.

Solinger, R. and Berghout, E. (1999), "The Goal/Question/Metric Method: a Practical Guide for Quality Improvement of Software Development”. McGraw-Hill.

Softex. (2007a), "MPS.BR General Guide" (versão 1.2). http:/www.softex.br/, June.

Softex. (2007b), "MPS.BR Implementation Guide", Part1: Level G; Part 2: Level F; Part 3: Level E; Part 4: Level D; Part 5: Level C; Part 6: Level B; Part 7: Level A. http://www.softex.br/portal/mpsbr/, June.

Schwaber, K. and Beedle, M. (2002), "Agile Software Development with Scrum'. Prentice Hall.

Turner, R. and Jain, A. (2002), "Agile Meets CMMI: culture Clash or Common Cause". XP/Agile Universe, p. 153-165. 